\title{
Investigation of the influence of the parameters of the VFH + method on the navigation efficiency of the mobile robot
}

\author{
ANDREJ ŠUTÝ, FRANTIŠEK DUCHOŇ \\ Institute of Robotics and Cybernetics \\ Slovak University of Technology \\ Ilkovičova 3, Bratislava \\ SLOVAK REPUBLIC
}

\begin{abstract}
The article focuses on verifying the effects of the VFH + navigation method parameters, proving to be very effective for the robot's reactive navigation. This research is based on our long-standing knowledge of histogram methods used in robot navigation. The article focuses on verifying the influence of crucial parameters - thresholds in a binary histogram, the $\mathrm{s}_{\max }$ parameter defining wide and narrow valleys, constants setting the criterion function, and the impact of robot dynamics on navigation. Many experiments were performed in a ROS simulation environment, and the article lists those significant confirming certain assumptions in setting these parameters.
\end{abstract}

Key-Words: $\mathrm{VFH}+$, parameters, mobile robot, ROS

Received: December 1, 2020. Revised: April 23, 2021. Accepted: May 10, 2021. Published: June 2, 2021.

\section{Introduction}

The navigation of a mobile robot is an essential task that it must solve for its successful deployment. The goal of navigation methods is to find optimal behavior that will ensure collision-free movement in the environment. In principle, we distinguish between local and global approaches. While the first look for solutions based on current perceptions (often called reactive navigation), the second usually works with a known representation of the environment. It looks for the optimal robot path (often called path planning).

At present, many local methods ensure the robot's collision-free movement. For example, the ROS framework offers local planners such as DWA (Dynamic-Window Approach) [1], FTC (Follow the Carrot) [2], or TEB (Timed Elastic Band) [3]. In practice, methods of reactive navigation are implemented, from the simplest ones as Bug Algorithms (0, 1, 2, 1 + 2, Tangent Bug) [4][5] to more complex ones such as Curvature Velocity Method (CVM)[6] or Nearness Diagram (ND) [7].

The direct comparison of these methods is known and available in the literature, e.g., in [8] Each of these methods provides various exciting features, such as considering the robot's dynamics or dimensions. However, not all of them are easily expandable and equally effective. Some fail in tight spaces or even in trap situations, unable to achieve a goal. This issue also appears in the methods implemented in ROS, the DWA method. It fails to control the influence of robot dynamics, and in some situations (Figure 1), it is inefficient navigation.

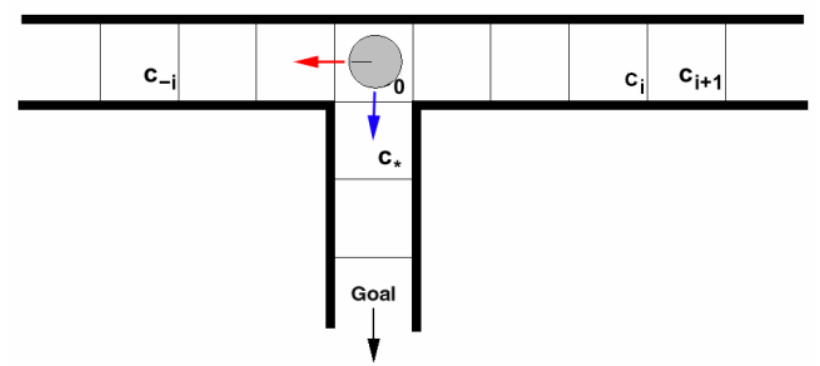

Figure 1: Situation where DWA local planning approach is inefficient. Red arrow - actual velocity of the robot, blue arrow - requested velocity of the robot based on DWA [9].

However, an influential group of methods can solve the robot's dimensions and dynamics, and these methods are very quickly extensible. They are called histogram methods which, in principle, allow easy extensibility by modifying the histograms themselves, as well as the criterion function that these methods use. The primary approach is the Vector Field Histogram (VFH) method [10], which can be extended with various modifications: VFH+ (dimensions of the robots, dynamics of the robot) [11], VFH* (partial planning) [12], MGTFH (traversability of the terrain) [13], VFH*TDT 
(dynamic obstacles avoidance), etc. A basic comparison of various modifications of these methods can be found in the articles [14] and [15]. A relevant study can be found in [16].

We consider these methods to be highly effective for the reasons mentioned above. Therefore, we decided to thoroughly investigate the influence of the basic parameters of the VFH+ method on the navigation efficiency of the mobile robot. We focused on the following essential parameters of the method: $\tau_{\text {high }}, \tau_{\text {low }}, s_{\max }, \mu_{1}, \mu_{2}, \mu_{3}$, and dynamic parameters of the robot $-v$ and $\omega$.

Our research provides a good overview of how the critical parameters of the VFH + method affect the efficiency of reactive navigation. As far as we know, such a comparison does not exist in the literature. The aim was to provide a good analysis of how to design these parameters and from which intervals to select these parameters. Of course, trial and error experiments can help set the parameters, but our research should provide a basic parameter design framework.

\section{VFH+ parameters}

The VFH+ method uses several stages of data reduction. One of them is a binary histogram $H^{b}$. This histogram was introduced to prevent oscillations in narrow corridors. This data reduction is based on the use of two thresholds, $\boldsymbol{\tau}_{\text {high }}$ and $\boldsymbol{\tau}_{\text {low }}$. The binary histogram is formed from a histogram $H^{p}$ based on the following:

$H_{k}^{b}=1 \quad$ if $H_{k}^{p}>\tau_{\text {high }}$

$H_{k}^{b}=0 \quad$ if $H_{k}^{p}<\tau_{\text {low }}$

$H_{k}^{b}=H_{k-1}^{b} \quad$ else

Based on the histograms created by the VFH + method, the output is a set of candidate valleys of the robot's future movement. The $\boldsymbol{s}_{\max }$ parameter specifies whether the candidate valley is wide or narrow. In the narrow candidate valley, there is one candidate direction, which is located in the middle of the valley:

$c_{n}=\frac{k_{r}+k_{l}}{2}$

Where $k_{r}$ and $k_{l}$ are free sectors on the right and left border of candidate valley. In the broad valley, there are two candidate directions:

$c_{r}=k_{r}+\frac{s_{\max }}{2}$

$c_{l}=k_{r}-\frac{s_{\max }}{2}$

If the direction to the target is between these candidates, this direction will also be included among the candidates. The set of selected directions then enter the selection using the criterion function:

$$
\begin{gathered}
g(c)=\mu_{1} \Delta\left(c, k_{t}\right)+\mu_{2} \Delta\left(c, \frac{\theta_{i}}{\varphi}\right) \\
+\mu_{3} \Delta\left(c, k_{n, i-1}\right) \\
\Delta(a, b)=\min \{|a-b|,|a-b-n|,|a-b+n|\}
\end{gathered}
$$

The first element of the function $\Delta\left(c, k_{t}\right)$ evaluates the distance between the candidate direction and the target direction. The second element $\Delta\left(c, \frac{\theta_{i}}{\varphi}\right)$ evaluates the difference between the candidate direction and the direction of rotation of the mobile robot's wheels. The third and last element $\Delta\left(c, k_{n, i-1}\right)$ evaluates the difference between the candidate direction and the previous best direction. In short, the first element ensures the selection of the path is closest to the target. Other factors ensure smooth movement; they serve as a short memory. Without these elements, situations could arise where a mobile robot oscillates between two sectors. The variables $\boldsymbol{\mu}_{1}, \boldsymbol{\mu}_{2}$, and $\boldsymbol{\mu} \boldsymbol{3}$ are used to set the character of the robot's resulting behavior. The best direction and thus the chosen direction of the robot is then the one that has the smallest value of the function. In general, to achieve the goal, it must apply [16]:

$\mu_{1} \geq \mu_{2}+\mu_{3}$

\section{Investigation of parameters}

An STDR simulator was used for the experiments. A differentially controlled circular robot's chassis was used for the simulation. Table 1 lists the basic parameters that were used in the $\mathrm{VFH}+$ simulations.

Table 1: VFH+ method - basic parameters

\begin{tabular}{ll}
\hline Cell size & $0.05[\mathrm{~m}]$ \\
\hline Active window size & $100[$ number of cells $]$ \\
\hline Number of sectors & 100 \\
\hline Radius of mobile robot & $0,3[\mathrm{~m}]$ \\
\hline $\begin{array}{l}\text { Safe distance from mobile } \\
\text { robot (C-obstacle) }\end{array}$ & $0,1[\mathrm{~m}]$ \\
\hline
\end{tabular}

To further set the VFH + method, the mobile robot was inserted between two obstacles (Figure 2), respectively, into the tunnel, and then other parameters were set (Table 2). The calibration was aimed so that the robot could not oscillate when passing through such an environment but could successfully move forward. 

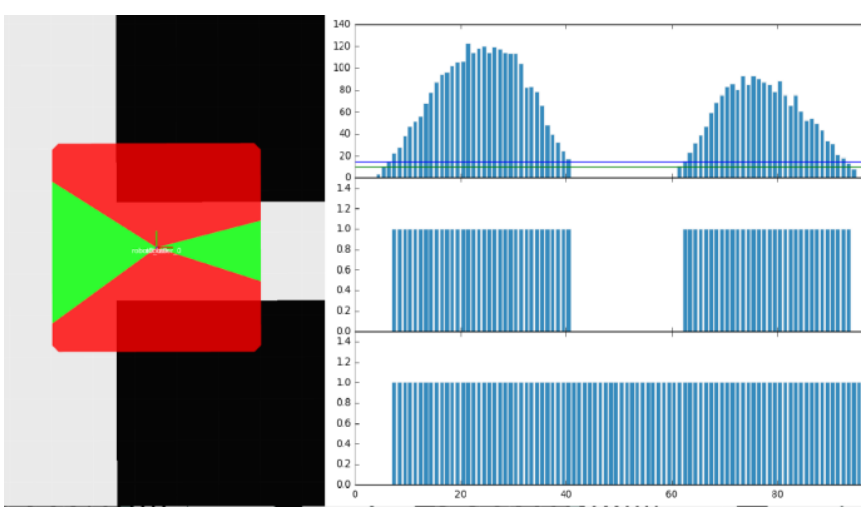

Figure 2: Output from the simulation - left; graphical representations of histograms used in the $\mathrm{VFH}+$ method - right (upper histogram shows the primary histogram $H^{p}$, the middle histogram shows the binary histogram $H^{b}$, the bottom histogram shows a histogram with a mask $H^{m}$ )

Table 2: Parameters specific for the $\mathrm{VFH}+$ method

\begin{tabular}{|c|c|}
\hline$s_{\max }$ & 20 \\
\hline$\tau_{\text {high }}$ & 20 \\
\hline$\tau_{\text {low }}$ & 10 \\
\hline$\mu_{1}$ & 5 \\
\hline$\mu_{2}$ & 2 \\
\hline$\mu_{3}$ & 2 \\
\hline $\begin{array}{ll}\text { Translational speed } \\
\text { limit }\end{array}$ & $0,5[\mathrm{~m} / \mathrm{s}]$ \\
\hline Rotational speed limit & $0,5[\mathrm{rad} / \mathrm{s}]$ \\
\hline
\end{tabular}

The influence of navigation efficiency parameters using the $\mathrm{VFH}+$ method was tested on several maps and under different conditions during the development. However, in this article, only significant ones are listed in the results.

The first tested parameters were the variables $\boldsymbol{\tau}_{\text {high }}$ and $\boldsymbol{\tau}_{\text {low }}$. These are the thresholds that determine the occupancy of the sector in the binary histogram $H^{b}$. This experiment aimed to test different combinations of these parameters and their effect on the robot's reactions. We expected that the higher the thresholds, the slower the reaction speed and the distances from the obstacles. At the same time, we wanted to test at what high values the collision would occur. In the experiment, we tested four different combinations $\tau_{\text {low }}=\{10 ; 20 ; 40 ; 10\}$ and $\tau_{\text {high }}=\{20 ; 40 ; 80 ; 50\}$.

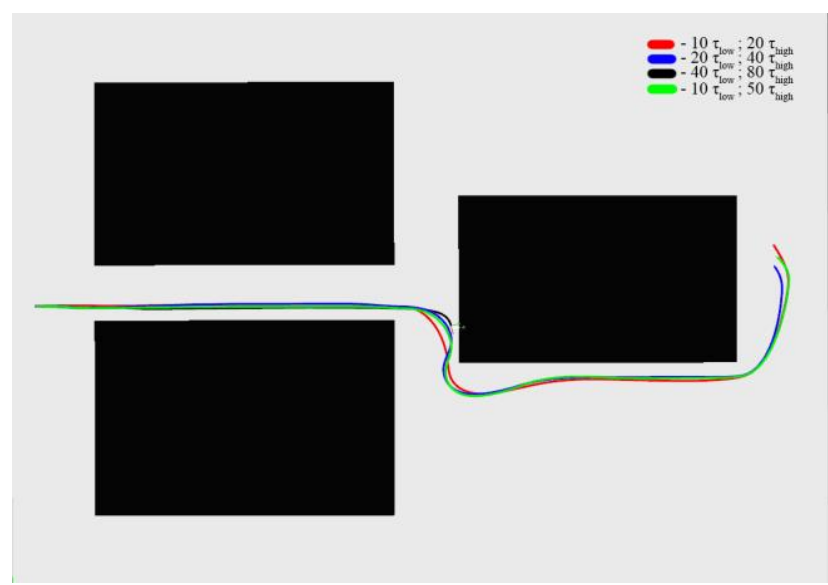

Figure 3: VFH+ method and the influence of parameters $\tau_{\text {high }}$ and $\tau_{\text {low. }}$

As expected, the higher the value of these variables, the closer the mobile robot had to go to the obstacle to respond to it (Figure 3). Three of the four attempts were positive, reaching the target. However, the combination $\tau_{\text {low }}=40 ; \tau_{\text {high }}=80$ did not reach the target, and a collision occurred. The method did not have enough time to react to the obstacle. If the thresholds are set too high and thus the frequency in the corresponding part of the histogram is not sufficient, the robot cannot respond to the obstacle. This is especially when there is not enough room to maneuver the robot - as in Figure 3, in front of the obstacle on the right. We remind that this is reactive navigation, where the robot perceives obstacles only within the measuring range of its sensors. Since the obstacle on the right "is noticed" at some distance from it, and the room for maneuver in front of it is minimal, there was a collision with the obstacle. Therefore, if we know that the robot will move in such narrow spaces, it is not appropriate to set the thresholds too high. To conclude, the variables $\tau_{\text {high }}$ and $\tau_{\text {low }}$ are critical in the configuration. At high values, a collision can occur.

Another test included the variable $s_{\max }$, which divides the valleys into wide and narrow and is also responsible for the obstacle's safe distance. Testing of this variable was divided into two experiments:

- circumventing one static obstacle,

- circumventing between several static obstacles.

In the first experiment, the mobile robot was placed in an environment with one static obstacle in the middle of the environment. The robot's goal was to circumvent this obstacle. If the configuration is correct, it will circle the target value without hitting an obstacle. Four values were chosen for the experiment, $s_{\max }=\{10 ; 20 ; 40 ; 80\}$. 


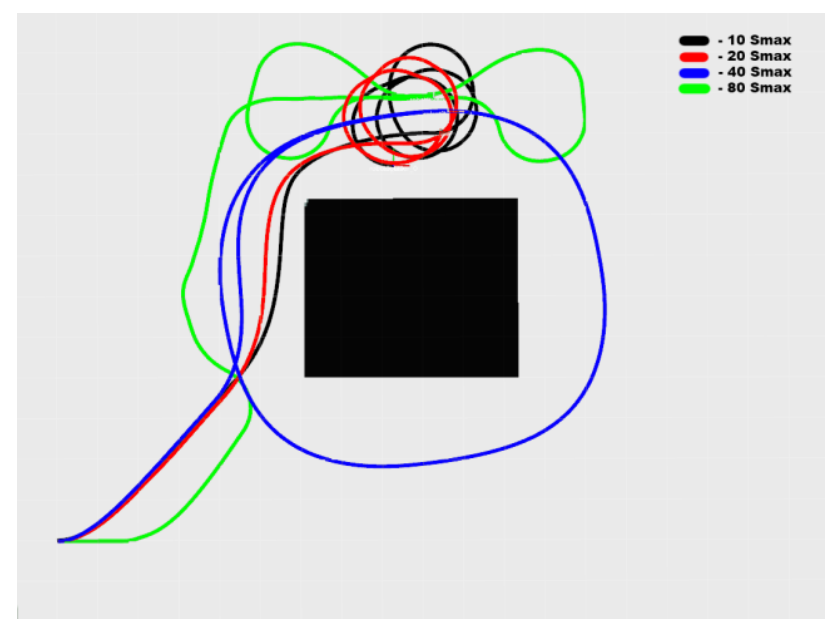

Figure 4: VFH+ method and the influence of parameter $\boldsymbol{s}_{\max }$ with one static obstacle.

It can be seen in Figure 4 that the values of 10 and 20 achieved good results. On the contrary, the worst value was 40 because it did not reach the target and instead revolved around the obstacle. The simulation with value 80 rotated at a greater distance from the target.

In the second part of the testing, it was necessary to move between several obstacles. This part of the experiment aimed to determine how the configuration would behave in this environment with the same values we used in the first experiment.

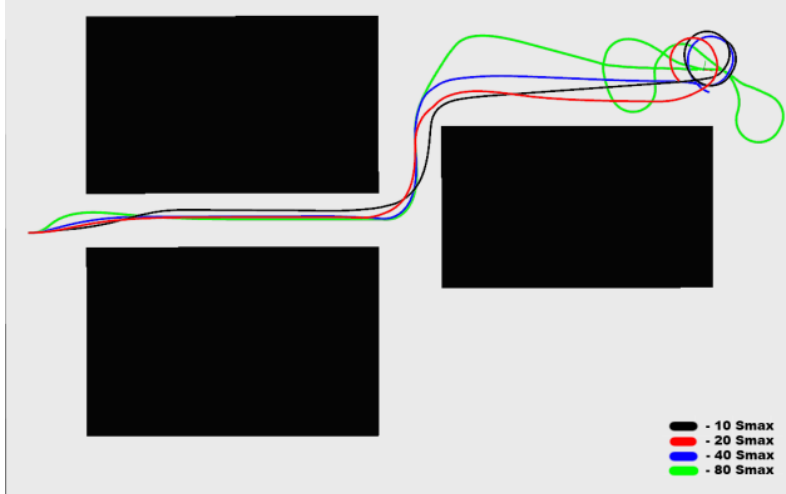

Figure 5: VFH+ method and the influence of parameter $\boldsymbol{s}_{\max }$ with several static obstacles.

The results show that the behavior of the robot is similar to the test with one static obstacle. In general, the lower the parameter $s_{\max }$ 's values, the smaller the distance from the obstacle robot keeps. On the contrary, too high a $s_{\max }$ value prevents the robot from reaching the target, especially when it is near the obstacle.

Another experiment was to test the coefficients of the criterion function. The experiment was performed in another environment, which more simulated the natural environment, i.e., the room-like environment. Some combinations of values were deliberately chosen in order not to comply with the condition (5). The combinations $\mu_{1}=\{1 ; 1 ; 1 ; 3 ; 4\}, \mu_{2}=\{1 ; 3 ; 1 ; 1 ; 2\}$, and $\mu_{3}=\{1$; $1 ; 3 ; 1 ; 2\}$ were chosen. Values that do not adhere to the condition (5) will most likely not reach the target area.

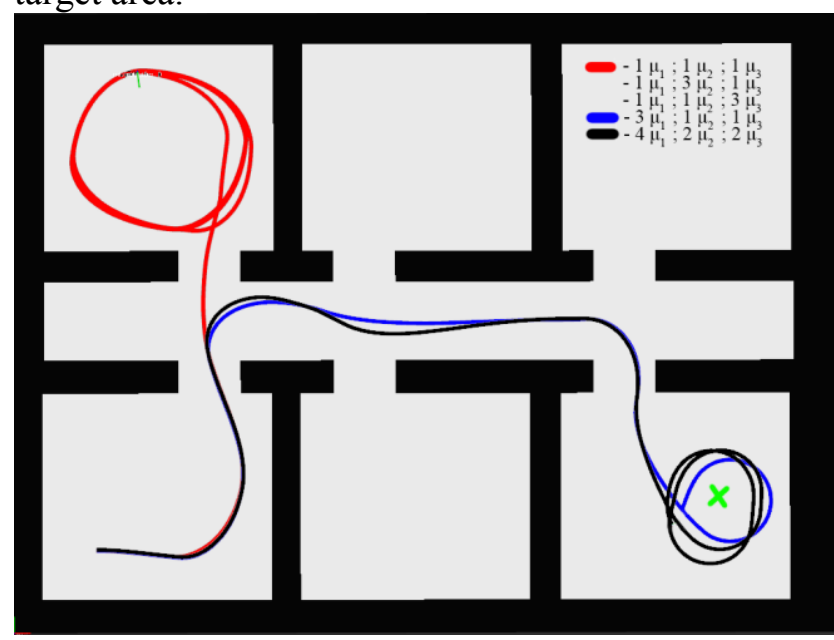

Figure 6: VFH+ method and the influence of the coefficients $\mu_{1}, \mu_{2}$, and $\mu_{3}$ in a room-like environment.

The result of the experiment is shown in Figure 6. The target area was the room located in the lower right corner. Of the five combinations tested, two were successful.

As can be seen, if condition (5) is not met, then the $\mathrm{VFH}+$ method cannot find the path to the target. In the experiments, we selected different combinations that did not satisfy condition $(5)-\left(\mu_{1} ; \mu 2 ; \mu 3\right)=$ $(1 ; 1 ; 1),(1 ; 3 ; 1),(1 ; 1 ; 3)$. In these cases, the mobile robot got into a room where it was impossible to getaway. The paths are drawn with a red line, and all three were very similar. The remaining two combinations $-\left(\mu_{1} ; \mu 2 ; \mu 3\right)=$ $(3 ; 1 ; 1),(4 ; 2 ; 2)$ led to the achievement of the goal. We have confirmed our assumption that condition (5) is decisive in selecting the target sectors. If their preference is to be goal-oriented, the requirement from the given equation must be applied. The first element of the function (4) is responsible for directing the robot as close to the target as possible. Thus, this factor's influence should be significantly greater than the other two, mainly considering the robot's movement's dynamics and fluidity. As can be seen in Figure 6, the red paths were very smooth and took into account the robot's dynamics, but they could not sufficiently direct the robot to the target in the part of the long corridor. 
The last experiment was to test the influence of dynamics on the VFH + navigation method. The dynamic properties of the robot for $\mathrm{VFH}+$ are crucial. It has a significant influence on the choice of direction. In the experiment, the values that limit the mobile robot's translational and rotational velocity were changed. By changing these values, the ICR changes fundamentally. When selecting test values, we chose three combinations $\mathrm{v}=\{1 ; 0.5 ; 1\}$ a $\omega=\{1 ; 1 ; 0.5\}$. The position of the mobile robot in each experiment started at the same location. We assumed that the ICR has a major influence in choosing the direction of a mobile robot. If this value is large, the traversability of the robot in a narrow space is reduced.

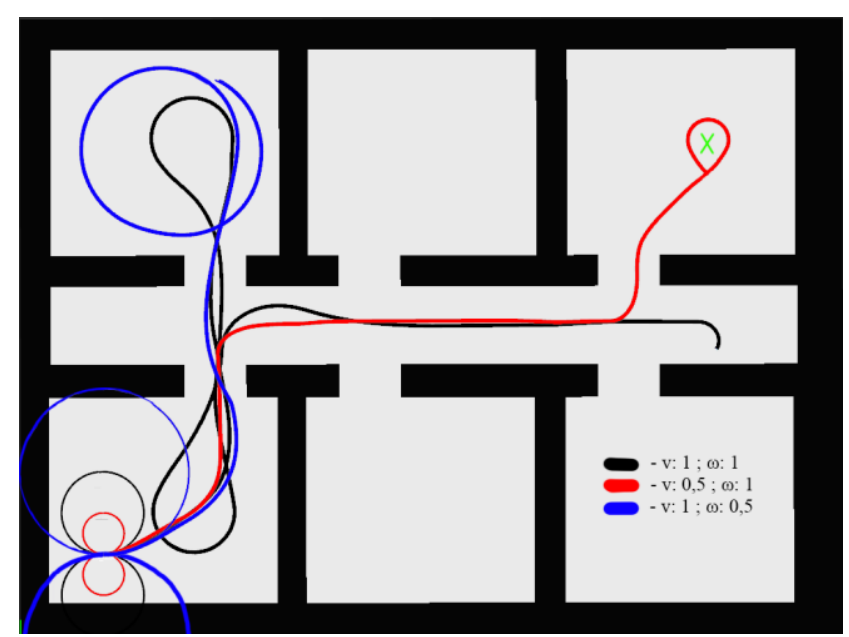

Figure 7: VFH+ method and the influence of the robot dynamics on navigation efficiency.

Figure 7 shows the result of the last test. The circles in the lower-left corner show the ICR point's size for a given combination of values. It illustrates the importance of selecting both variables. Only one of the tested combinations reached the target. Another did not reach the target. The high dynamics made it impossible to choose the sectors that led to the target area. The last combination did not reach the target area. For reasons similar to the previous combination, it failed to turn in a narrow space where a collision with an obstacle occurred.

In our experiments, we demonstrated the effect of setting the parameters of the VFH + method on the efficiency of the reactive navigation itself. We performed the experiments in a simulation environment in ROS. Despite the simplifications used mainly in modeling the measurement of sensors, we managed to create a methodology for correctly setting the parameters of the VFH + method. Using the knowledge we have created, other researchers do not have to perform many experiments in trial and error but can rely on the results we have achieved. Thus, their work can be much more efficient in setting up this reactive navigation.

\section{Conclusion}

This research aimed to examine the possibility of reactive navigation method $\mathrm{VFH}+$ and performance the evaluation under various conditions and configurations in the ROS environment. We concluded that the chosen VFH + method is very reliable at well-chosen configuration values in most cases. Since we tested this method on a simulator and not on a real device, we made some compromises. In natural conditions, the sensors contain more significant errors when measuring more distant obstacles. Typically used sensors in reactive navigation are active sensors, e.g., LIDARs that send energy into space. To get information about the location of obstacles, they wait for an echo from the obstacles. If these obstacles are too far apart due to the transmitted signal's nature, more significant errors occur than nearby obstacles. We proved this, for example, in the article [18], where we dealt with the probabilistic model of the Hokuyo UTM-30LX sensor. However, in the experiments in this article, our goal was not to create an exact model of the sensor but to demonstrate the influence of the parameters of the VFH + method on the efficiency of reactive navigation. Of course, the method uses such sensors. However, to focus sufficiently on the parameters, we artificially reduced the measuring range of such a sensor rather than using exact probabilistic models, such as we derived in the article [18].

Of course, the VFH + method depends on a reliable self-localization of the robot (e.g., SLAM). If this module fails, VFH + cannot be used. In our experiments, we proceeded from the essence that such a module is $100 \%$ functional, and we have reliable information about the position of the robot at all times. The reactive navigation method ( $\mathrm{VFH}+$ ) should also be combined with the path planning module so that the overall navigation is highly efficient and optimal in a known environment. In our experiments, we did not consider path planning and therefore not even a priori knowledge of the environment. Only reactive navigation is used in the experiments, i.e., the robot knew only about its surroundings regarding the sensors' measuring range. Likewise, the VFH + method is not suitable for circumventing moving obstacles, as we have already shown in our modification of this method described in Articles [14] and [15]. In many 
experiments with this method, we set the parameters of the method by trial and error. Therefore, we wanted to create at least a framework for setting parameters so that it is possible to set the method effectively at the very beginning.

The whole source code can be found here: https://github.com/legenda971/VFH

\section{References:}

[1] dwa_local_planner:

http://wiki.ros.org/dwa_local_planner

[2] asr_ftc_local_planner:

http://wiki.ros.org/asr_ftc_local_planner

[3] teb_local_planner:

http://wiki.ros.org/teb_local_planner

[4] E. Plaku, "Algorithms for Sensorbased Robotics, Lecture II, III: Bug Path-Planning Algorithms," Department of Computer Science, Laboratory for Compuatational Sensing and Robotics, John Hopkins University, 2010.

[5] H. Choset et al., "Principles of Robot Motion (Theory, Algorithms, and Implementations)," Boston: MIT Press, 2005.

[6] R. Simmons, "The Curvature-Velocity Method for Local Obstacle Avoidance," International Conference on Robotics and Automation, Pittsburgh, PA, 1996.

[7] J. Minguez and L. Montano, "Nearness Diagram Navigation (ND) (A New Real Time Collision Avoidance Approach)," Proceedings of the IEEE/RSJ International Conference on Intelligent Robots and Systems, Zaragoza, 2000.

[8] R. Siegwart, I. R. Nourbakhsh and D. Scaramuzza, "Introduction to autonomous mobile robots," MIT press, 2011.

[9] W. Burgard, "Introduction to Mobile Robotics: Path and Motion Planning", ais.informatik.uni-

freiburg.de/teaching/ss18/robotics/slides/19pathplanning-long.pdf

[10] J. Borenstein and Y. Koren, "The Vector Field Histogram - Fast Obstacle Avoidance For Mobile Robots," IEEE Journal of
Robotics and Automation, vol. 7, no. 3, pp. 278-288, 1991.

[11] I. Ulrich and J. Borenstein, "VFH+: Reliable Obstacle Avoidance for Fast Mobile Robots," Proceedings of the IEEE International Conference of Robotics and Automation, Leuven, Belgium, 1998.

[12] I. Ulrich and J. Borenstein, "VFH*: Local Obstacle Avoidance with Look-Ahead Verification," Proceedings of the IEEE International Conference of Robotics and Automation, San Francisco, CA, 2000.

C. Ye, "Navigating a mobile robot by a traversability field histogram," IEEE Transactions on Systems, Man, and Cybernetics, vol. 37, no. 2, pp. 361-372, 2007.

[14] A. Babinec et al. "VFH* TDT (VFH* with Time Dependent Tree): A new laser rangefinder based obstacle avoidance method designed for environment with nonstatic obstacles," Robotics and autonomous systems, vol. 62, no. 8, pp. 1098-1115, 2014.

[15] A. Babinec et al., "Vector Field Histogram* with look-ahead tree extension dependent on time variable environment," Transactions of the Institute of Measurement and Control, vol. 40, no. 4, pp. 1250-1264, 2018.

[16] I. Ulrich and J. Borenstein, "VFH+: Reliable Obstacle Avoidance for Fast Mobile Robots, http://www.cs.cmu.edu/ iwan/papers/vfh+. pdf.

[17] Burak Kaan Özbek, Metin Turan, Research on the Availability of VINS-Mono and ORB-SLAM3 Algorithms for Aviation, WSEAS Transactions on Computers, ISSN / E-ISSN: 1109-2750 / 2224-2872, Volume 19, 2020, Art. \#27, pp. 216-223.

[18] Dekan, M., Duchon̆, F., Babinec, A., Hubinský, P., Kajan, M., \& Szabova, M. (2015). Versatile approach to probabilistic modeling of Hokuyo UTM-30LX. IEEE Sensors Journal, 16(6), 1814-1828. 
Contribution of individual authors to the creation of a scientific article (ghostwriting policy)

Andrej Šutý carried out the simulation and the results verifications.

František Duchoň was responsible for the conceptualization, supervision, and writing the article.

Sources of funding for research presented in a scientific article or scientific article itself

This work was supported by grants VEGA 1/0754/19 and VEGA 1/0775/20.

\section{Creative Commons Attribution}

\section{License 4.0 (Attribution 4.0}

International , CC BY 4.0)

This article is published under the terms of the Creative Commons Attribution License 4.0

https://creativecommons.org/licenses/by/4.0/deed.en $\underline{\underline{U S}}$ 